\title{
Progression of Esophageal Dysmotility in Systemic Sclerosis
}

\author{
JOHN VISCHIO, FAHD SAEED, MOZAFAREDDIN KARIMEDDINI, ASIA MUBASHIR, RICHARD FEINN, \\ GLORIA CALDITO, KIM STRIEGEL, and NAOMI ROTHFIELD
}

\begin{abstract}
Objective. To longitudinally evaluate esophageal dysmotility (ED) in patients with limited cutaneous systemic sclerosis (lcSSc) and diffuse cutaneous systemic sclerosis (dcSSc).

Methods. We performed a retrospective review of all adult patients with SSc seen between 1995 and 2008. Patients were included if they had undergone 2 or more esophageal transit scintigraphy (ETS) studies at least 1 year apart. Data from 382 ETS studies of 102 patients with SSc were analyzed. Eighty patients had $\mathrm{lcSSc}$ and 22 patients had dcSSc. A grading system was used to quantify the degree of esophageal dysfunction, ranging from grade 0 (normal) to grade 3 (severe hypomotility). Change in esophageal motility over time was evaluated and compared between the limited and diffuse subtypes.

Results. Sixty-eight patients $(66.7 \%)$ had an abnormal ETS study at any time. Of patients with dcSSc, $95.4 \%$ had an abnormal ETS study, compared to $58.5 \%$ of patients with lcSSc. dcSSc and regurgitation were independent risk factors for ED. There was no association between the presence of anticentromere antibodies or antitopoisomerase (anti-Scl-70) antibodies and an abnormal ETS study. Esophageal motility in patients with dcSSc worsened in $96 \%$ of cases compared with only $58.8 \%$ in those with lcSSc.

Conclusion. ED is more frequent in patients with dcSSc than in those with lcSSc, and is more likely to deteriorate over time. Given the potential associated risks of erosive esophagitis, Barrett's esophagus, and esophageal cancer in patients with SSc, routine screening and monitoring for ED is advised. (First Release March 1 2012; J Rheumatol 2012;39:986-91; doi:10.3899/jrheum.110923)
\end{abstract}

Key Indexing Terms:

SYSTEMIC SCLEROSIS

ESOPHAGEAL DYSMOTILITY

ESOPHAGEAL TRANSIT SCINTIGRAPHY

Systemic sclerosis (SSc) is a chronic, multisystem autoimmune disorder manifested by excessive deposition of connective tissue in the skin and viscera. The inflammation, vascular dysfunction, and excessive fibrosis characteristic

From the Department of Medicine, Division of Rheumatology, Department of Psychiatry and Biostatistics Center, and the Department of Radiology,

University of Connecticut Health Center, Farmington, Connecticut; Department of Medicine, Division of Rheumatology, Department of Biometry, Louisiana State University Health Sciences Center, New Orleans, Louisiana; and Department of Medicine, Howard University Hospital, Washington, DC, USA.

J. Vischio, MD, Fellow, Department of Medicine, University of Connecticut Health Center; F. Saeed, MD, Fellow, Department of Medicine/Rheumatology, Louisiana State University Health Sciences Center; M. Karimeddini, MD, Associate Professor, Department of Medicine, University of Connecticut Health Center; A. Mubashir, MD, Assistant Professor, Department of Medicine, Howard University Hospital; R. Feinn, PhD, Assistant Professor, Department of Psychiatry, University of Connecticut Health Center; G. Caldito, PhD, Associate Professor, Department of Biometry, Louisiana State University Health Sciences Center; K. Striegel, APRN, Department of Medicine, University of Connecticut Health Center; N. Rothfield, MD, Professor of Medicine, Department of Medicine, University of Connecticut Health Center.

Dr. Vischio and Dr. Saeed contributed equally to this report.

Address correspondence to Dr. N. Rothfield, University of Connecticut Health Center, Division of Rheumatology, MC 5353, 263 Farmington Ave., Farmington, CT 06030, USA. E-mail: Rothfield@nso.uchc.edu Accepted for publication December 16, 2011. of both limited cutaneous SSc (lcSSc) and diffuse cutaneous SSc (dcSSc) commonly involve the gastrointestinal (GI) tract $^{1}$. Esophageal function is affected in up to $90 \%$ of patients with $\mathrm{SSc}^{1,2,3,4}$. Symptoms of esophageal dysfunction may include dyspepsia, dysphagia, nausea, or vomiting, which can lead to weight loss, chronic cough, hoarse voice, pharyngitis, laryngospasm, asthma, and recurrent aspiration. Symptoms, however, may be unreliable in estimating the presence and extent of esophageal disease in $\mathrm{SSc}^{5}$. Early diagnosis is important to reduce the risk of complications ${ }^{6}$.

Esophageal dysfunction in SSc typically occurs within the lower two-thirds of the esophagus as a weakened lower esophageal sphincter (LES) and loss of peristalsis, allowing pathological reflux of acidic gastric contents into the esophagus $^{6,7}$. Gastroesophageal reflux disease (GERD) may then lead to erosive esophagitis with ulceration, strictures, and fistulae. As a result, patients with SSc are at risk of developing Barrett's esophagus and esophageal cancer ${ }^{7,8}$. Routine screening for esophageal dysfunction may be included in the initial evaluation of patients with SSc.

Esophageal transit scintigraphy (ETS) has been shown to be as effective as esophageal manometry in screening patients with esophageal motility disorders ${ }^{9}$. Using esophageal manometry as the "gold standard," sensitivities

Personal non-commercial use only. The Journal of Rheumatology Copyright @ 2012 . All rights reserved. 
and specificities up to $95 \%$ and $96 \%$, respectively, have been reported ${ }^{10,11}$. As an indicator of esophageal dysmotility (ED) in both early and advanced SSc, ETS has a higher sensitivity than manometry and barium swallows $5,12,13,14,15$. In patients with SSc, ETS is easy to perform, and yields quantitative information suitable for serial studies to monitor disease progression and treatment efficacy.

Given the limited data evaluating the progression of ED in patients with $\mathrm{SSc}^{16,17}$, the objective of our study was to longitudinally evaluate esophageal motility and risk factors for progression of ED in patients with lcSSc and dcSSc.

\section{MATERIALS AND METHODS}

Patients. A total of 382 ETS studies from 102 patients with SSc based on the American Rheumatism Association diagnostic criteria ${ }^{18}$ were retrospectively reviewed. Every patient with SSc, regardless of whether they had any GI symptoms, had an ETS study ordered on their first visit and yearly thereafter. All patients had 2 or more ETS studies performed at least 1 year apart between January 1995 and December 2008. There were 91 women and 11 men, with a mean age of 51.6 years (range 19-80 yrs) and a mean disease duration of $6.9 \pm 5.0$ years from their first episode of Raynaud's phenomenon (Table 1). At the time of the first ETS study, the classification into dcSSc and lcSSc types was based on distribution of skin thickness using the modified Rodnan skin score (MRSS) ${ }^{19}$, the diffuse cutaneous SSc type with skin tightness involving the trunk and/or proximal extremities. The mean MRSS was $10.4 \pm 11.9$. Eighty patients had lcSSc and 22 patients had dcSSc. At the time of their initial visit, each patient was tested for antibodies, including anticentromere antibodies (ACA) and anti-Scl-70 antibodies. Thirty-five patients (34.3\%) were ACA-positive and $12(11.8 \%)$ were anti-Scl-70 antibody-positive. At the time of their baseline ETS study, 56 patients (54.9\%) had heartburn, 42 (41.2\%) had regurgitation (defined by the patient-reported return of swallowed food into the mouth), and $6(5.9 \%)$ had dysphagia. At any point during the period in which the patients were followed, 72 patients (70.5\%) had heartburn, $58(56.8 \%)$ had regurgitation, and 25 (24.5\%) had dysphagia.

ETS. A 2-part evaluation using ETS was conducted in each patient, in both the upright (sitting) and supine positions. For each study, $0.25 \mathrm{mCi}$ of ${ }^{99 \mathrm{~m}} \mathrm{Tc}$ diethylene-triamine-pentaacetic acid was mixed with $10 \mathrm{ml}$ of water. Patients arrived fasting for this study, and were instructed to take the radioactive bolus in their mouth and hold it until instructed to swallow in 1 gulp. A large-field-of-view gamma camera was used, and sequential $64 \times$ 64 images were acquired in the anterior projection at 1 frame/s. Images were stored in a dedicated computer system. Data were analyzed using standard nuclear medicine software for generating time/activity curves from dynamic studies. Regions of interest were drawn for the upper, middle, and lower thirds of the esophagus. The time/activity curve from the upper esophagus was used as the control in assessing the integrity of the radioactive bolus: 1 bolus versus a fragmented bolus. A fragmented bolus invalidated the study. Interpretation was performed by 1 radiologist and was based on visual evaluation of time/activity curves derived from the middle and distal thirds of the esophagus. A study was considered normal (Grade 0) when there was more than 90\% emptying of the esophagus in < $15 \mathrm{~s}$. Esophageal transit time was considered mildly delayed (Grade 1) when 50\%-90\% emptying occurred in $30 \mathrm{~s}$, moderate (Grade 2) when $50 \%-90 \%$ emptying occurred in $60 \mathrm{~s}$, and severe (Grade 3) when $<50 \%$ emptying occurred in $60 \mathrm{~s}$.

A patient was determined to have ED if any portion (upright proximal, upright distal, supine proximal, or supine distal) of the ETS revealed dysmotility. Progression of ED was observed and defined as worsening in severity of ED at either the proximal and/or distal esophagus by the end of the patient's followup period.

Statistical analysis. Chi-square and Fisher's exact test were used to compare ED rates between the various subgroups. The Wilcoxon rank-sum test was used to analyze the relationship between the number of ETS studies performed, length of followup, MRSS, and ED. Multiple logistic regression analysis was used to assess independent risk factors for ED. A p value < 0.05 was considered significant for all statistical tests.

Table 1. Summary of patient characteristics. Data are n (\%) unless otherwise indicated.

\begin{tabular}{lccc}
\hline Characteristic & All Patients, $\mathrm{n}=102$ & $\mathrm{lcSSc}, \mathrm{n}=80(78.4 \%)$ & $\mathrm{dcSSc}, \mathrm{n}=22(21.6 \%)$ \\
\hline Mean age, yrs (range) & $51.6(19-80)$ & $52.3(19-80)$ & $49.0(26-74)$ \\
Mean disease duration, yrs (range) & $6.9(2-25)$ & $6.8(2-24)$ & $7.0(2-25)$ \\
Women & $91(89.2)$ & $76(83.5)$ & $15(16.5)$ \\
Men & $11(10.8)$ & $4(36.3)$ & $7(63.4)$ \\
White & $89(87.3)$ & 74 & 15 \\
African American & $4(3.9)$ & 2 & 2 \\
Hispanic & $8(7.8)$ & 6 & 2 \\
Asian & $1(1.0)$ & 0 & $1(4.5)$ \\
Mean no. ETS studies (range) & $3.7(2-8)$ & $3.7(2-8)$ & $4.0(2-8)$ \\
Mean no. yrs followed (range) & $5.11(2-18)$ & $5.02(2-18)$ & $5.45(2-9)$ \\
ACA-positive & $35(34.3)$ & $34(42.5)$ & $1(4.6)$ \\
Scl-70-antibody-positive & $12(11.8)$ & $5(6.2)$ & $7(31.8)$ \\
Mean MRSS, \% (range) & $10.4(0-55)$ & $7.6(0-55)$ & $19.1(0-41)$ \\
Heartburn at initial ETS & $56(54.9)$ & $42(52.5)$ & $14(63.6)$ \\
Heartburn at any time & $71(69.6)$ & $54(67.5)$ & $17(77.3)$ \\
Regurgitation at initial ETS & $42(41.2)$ & $32(40.0)$ & $10(45.4)$ \\
Regurgitation at any time & $58(56.9)$ & $45(56.2)$ & $13(59.1)$ \\
Dysphagia at initial ETS & $6(5.9)$ & $5(6.2)$ & $1(4.5)$ \\
Dysphagia at any time & $25(24.5)$ & $19(23.8)$ & $6(27.3)$ \\
Antireflux medication & $71(69.6)$ & $53(66.3)$ & $18(81.8)$ \\
\hline
\end{tabular}

lcSSc: limited cutaneous systemic sclerosis; dcSSc: diffuse cutaneous systemic sclerosis; ETS: esophageal transit scintigraphy; ACA: anticentromere antibodies; MRSS: modified Rodnan skin score. 


\section{RESULTS}

The number of ETS studies performed was not significantly associated with the presence of $\mathrm{ED}$. Mean $\pm \mathrm{SD}$, median, and range for number of ETS studies were similar for patients with and without ED (3.9 $\pm 1.8,3.0,2$ to 8 ; and 3.4 $\pm 1.8,3.0,2$ to 8 , respectively; $\mathrm{p}=0.08$ ). Sixty-eight patients $(66.7 \%)$ had an abnormal ETS study at any time. A significantly higher proportion of patients with dcSSc had an abnormal ETS study compared to those with lcSSc (95.4\% vs $58.8 \%$, respectively; $p<0.01$; Table 2). The mean MRSS in patients with an abnormal ETS study was greater (12.5 \pm 12.4, range 0-55) for those patients with a normal ETS study $(5.9 \pm 9.5$, range $0-35.3 ; \mathrm{p}<0.01)$. There was no association between the presence of ACA or Scl-70 antibodies and an abnormal ETS study. Thirty-four of the 80 patients with $1 \mathrm{cSSc}$ were ACA-positive. Of those, 20 (58.8\%) had an abnormal ETS study compared with $58.7 \%$ of the patients who were ACA-negative $(p>0.99)$. Seven of the 22 patients with dcSSc were Scl-70-positive. All 7 had an abnormal

Table 2. Comparison of esophageal dysmotility rates. Data are $\mathrm{n}(\%)$.

\begin{tabular}{|c|c|c|}
\hline Characteristic & Esophageal Dysmotility, n (\%) & $\mathrm{p}$ \\
\hline $\mathrm{dcSSc}, \mathrm{n}=22$ & $21(95.4)$ & \\
\hline $1 \mathrm{cSSc}, \mathrm{n}=80$ & $47(58.8)$ & $<0.01 * *$ \\
\hline \multicolumn{3}{|l|}{ ACA test } \\
\hline Positive, $\mathrm{n}=35$ & $21(60.0)$ & \\
\hline Negative, $\mathrm{n}=67$ & $47(70.2)$ & 0.30 \\
\hline \multicolumn{3}{|l|}{ Scl-70 test } \\
\hline Positive, $\mathrm{n}=12$ & $10(83.3)$ & 0.33 \\
\hline Negative, $\mathrm{n}=90$ & $58(64.4)$ & \\
\hline \multicolumn{3}{|l|}{ Heartburn (baseline) } \\
\hline Yes, $\mathrm{n}=56$ & $42(75.4)$ & $0.049 *$ \\
\hline No, $n=46$ & $26(56.5)$ & \\
\hline \multicolumn{3}{|l|}{ Heartburn (any time) } \\
\hline Yes, $\mathrm{n}=71$ & $53(74.6)$ & $<0.01 * *$ \\
\hline No, $n=31$ & $15(48.4)$ & \\
\hline \multicolumn{3}{|l|}{ Regurg (baseline) } \\
\hline Yes, $n=42$ & $31(73.8)$ & 0.20 \\
\hline No, $n=60$ & $37(61.7)$ & \\
\hline \multicolumn{3}{|l|}{ Regurg (any time) } \\
\hline Yes, $\mathrm{n}=58$ & $47(81.0)$ & $<0.01 * *$ \\
\hline No, $n=44$ & $21(47.7)$ & \\
\hline \multicolumn{3}{|l|}{ Antireflux therapy } \\
\hline Yes, $\mathrm{n}=71$ & $55(77.5)$ & $<0.01 * *$ \\
\hline No, $n=31$ & $13(41.9)$ & \\
\hline \multicolumn{3}{|l|}{ Sex } \\
\hline Women, $\mathrm{n}=91$ & $59(64.8)$ & 0.33 \\
\hline Men, $\mathrm{n}=11$ & $9(81.8)$ & \\
\hline \multicolumn{3}{|l|}{ Baseline dysphagia } \\
\hline Yes, $n=6$ & $5(83.3)$ & \\
\hline No, $n=96$ & $63(65.6)$ & 0.66 \\
\hline \multicolumn{3}{|l|}{ Dysphagia any time } \\
\hline Yes, $\mathrm{n}=6$ & $20(80.0)$ & \\
\hline No, $n=96$ & $48(62.3)$ & 0.10 \\
\hline
\end{tabular}

* Significant at $5 \%$ level $(0.01<\mathrm{p}<0.05)$. ** Significant at $1 \%$ level $(\mathrm{p}<$ $0.01)$. lcSSc: limited cutaneous systemic sclerosis; dcSSc: diffuse cutaneous systemic sclerosis; ACA: anticentromere antibodies.
ETS study, compared with $93.3 \%$ who were Scl-70-negative ( $p>0.99$ ). An abnormal ETS study was associated with the presence of heartburn at any time $(\mathrm{p}<0.01)$ and regurgitation at any time $(\mathrm{p}<0.01)$, but not with dysphagia $(\mathrm{p}=0.1)$. Fifty-five $(77.5 \%)$ patients taking antireflux medication had an abnormal ETS study, compared with 13 (41.9\%) patients who were not $(\mathrm{p}<0.01)$.

Using separate univariate analyses, patients with dcSSc, higher MRSS, the presence of heartburn or regurgitation at any time, and the use of antireflux medication were significantly associated with an abnormal ETS study. However, using multivariate analysis with a multiple logistic regression model, dcSSc and regurgitation at any time were the only independent findings that were significantly associated with an abnormal ETS study. Adjusted odds for an abnormal ETS study among patients with dcSSc were 12.7 times greater (95\% CI 1.4-111.1) than for those patients with lcSSc $(\mathrm{p}=0.02)$. Adjusted odds for an abnormal ETS study among patients with regurgitation at any time during followup were 5.8 times greater (95\% CI 1.8-18.9) than for patients without regurgitation $(\mathrm{p}=0.04)$.

Serial ETS studies were evaluated for progression of ED. Twenty-one patients (95.5\%) with dcSSc had worsening ED on a subsequent ETS study (mean followup $=7.0$ years), 1 patient $(0.5 \%)$ remained unchanged, and none improved. In contrast, 47 patients (58.8\%) with lcSSc had worsening ED on a subsequent ETS study (mean followup $=6.8$ years), 15 $(18.8 \%)$ remained unchanged, and $18(22.5 \%)$ had an improved esophageal transit time on a subsequent ETS study (Table 3). Adjusted odds for a worsening ETS study (i.e., ED) among patients with dcSSc were 12.7 times the odds for patients with lcSSc (OR 12.7, 95\% CI 1.4-111.1, $\mathrm{p}=0.02$ ). Stacked bar graphs illustrating progression of ED in patients with lcSSc and dcSSc are shown in Figure 1. Figure 1 illustrates that the number of patients who obtained serial ETS studies declined over time. However, the proportion of patients with more severe ED increased over time, particularly in the patients with dcSSc.

\section{DISCUSSION}

The GI tract is affected in 75\%-90\% of patients with SSc, with the esophagus being the most frequently involved portion $^{1,2,3,4,20}$. Although studies have shown that up to $90 \%$ of patients with SSc have ED, many of these patients are asymptomatic $^{4,5,21}$. In our study, the proportion of patients

Table 3. Change in ETS studies over time.

\begin{tabular}{lccc}
\hline Type of SSc & $\begin{array}{c}\text { Worsened, } \\
\mathrm{n}(\%)\end{array}$ & $\begin{array}{c}\text { Unchanged, } \\
\mathrm{n}(\%)\end{array}$ & $\begin{array}{c}\text { Improved, } \\
\mathrm{n}(\%)\end{array}$ \\
\hline $\begin{array}{l}\mathrm{lcSSc}, \mathrm{n}=80 \\
\mathrm{dcSSc}, \mathrm{n}=22\end{array}$ & $\begin{array}{c}47(58.7) \\
21(95.5)\end{array}$ & $\begin{array}{c}15(18.8) \\
1(0.5)\end{array}$ & $\begin{array}{c}18(22.5) \\
0\end{array}$ \\
\hline
\end{tabular}

ETS: esophageal transit scintigraphy; lcSSc: limited cutaneous systemic sclerosis; dcSSc: diffuse cutaneous systemic sclerosis. 


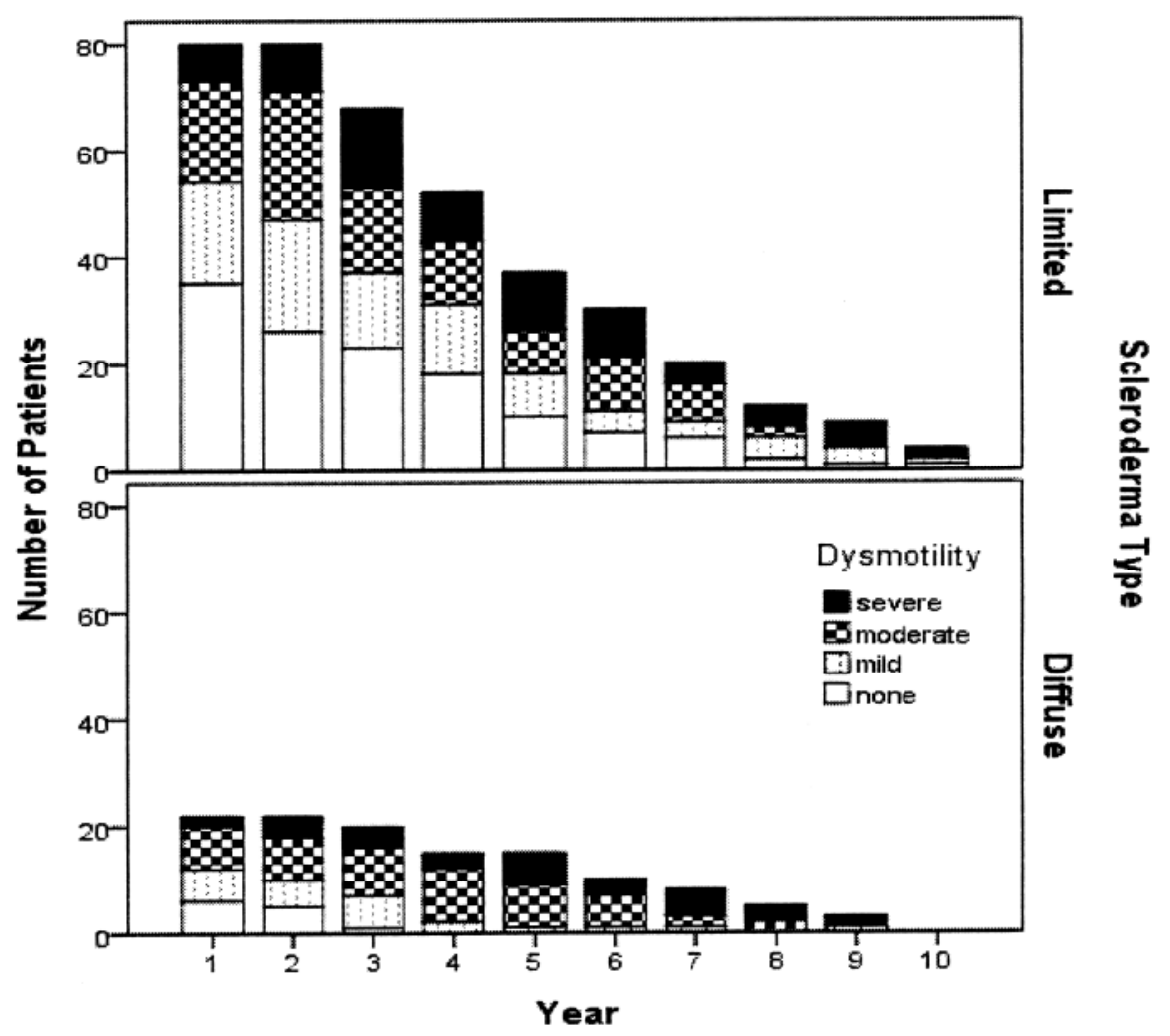

Figure 1. Progression of esophageal dysmotility over time in limited cutaneous systemic sclerosis and diffuse cutaneous systemic sclerosis.

with ED by ETS was found to be $66.7 \%$. Common esophageal manifestations in SSc include motility abnormalities, GERD, Barrett's esophagus, adenocarcinoma, infectious esophagitis, and drug-induced esophagitis, with complications of GERD also including erosive esophagitis, bleeding, and stricture formation ${ }^{20}$. Patients with SSc who develop ED may develop early satiety, regurgitation of food, progressive weight loss, malnutrition, or impaction of food. In addition, ED in patients with SSc may correlate with the presence of interstitial lung disease (ILD) ${ }^{22}$. Proposed progression of GI involvement in SSc includes vascular damage (grade 0), neurogenic impairment (grade 1), and myogenic dysfunction, with the replacement of normal smooth muscle by collagenous fibrosis and atrophy (grade 2$)^{2}$.

In accordance with previous published studies, we found that ED was more frequent and severe in patients with dcSSc than in those with $1 c S S c$ 15,22,23,24,25,26. Those studies also demonstrated that ED was more frequent and severe in patients who are anti-Scl-70 antibody-positive and ACA-negative. Interestingly, however, and in contrast to these data, we found that in our patient population there was no association between the presence of anti-Scl-70 antibody or ACA and the finding of an abnormal ETS study. Multivariate analysis revealed that in addition to having diffuse subtype, the symptom of regurgitation was the only independent finding that was significantly associated with ED. Our patients who were taking antireflux medication were more likely to have an abnormal ETS study, suggesting that these patients were more symptomatic and had more severe esophageal involvement. A recent study by Pakozdi, et al demonstrated that although longterm therapy with lansoprazole aided in reducing gastroesophageal symptoms in patients with SSc, there was no beneficial effect in preventing the progression of $\mathrm{ED}^{27}$.

Prior to our study, there were limited published data evaluating the progression of esophageal dysfunction in patients with SSc. Baron and Arzoumanian longitudinally followed 19 patients with progressive SSc by ETS over 3 to 5 years. They noted a gradual deterioration of esophageal transit at both $20 \mathrm{~s}$ and $10 \mathrm{~min}$. Deterioration occurred in $58.5 \%$ of followup ETS studies in patients at $20 \mathrm{~s}$, and in $48 \%$ of patients at $10 \mathrm{~min}^{16}$. Dantas, et al studied the progression of esophageal involvement by manometry on 2 occasions with a median interval of 40 months in 17 females with SSc compared to 14 healthy females ${ }^{17}$. The LES pressure and amplitude of contractions in the esophageal body were lower in patients with SSc than in controls. In 16 of the 17 patients with SSc, no difference in LES pressure, esophageal contraction amplitude, duration, or velocity was observed between the first and second manometric evaluation. In only

Personal non-commercial use only. The Journal of Rheumatology Copyright @ 2012 . All rights reserved. 
1 patient with SSc, the distal esophageal contractions deteriorated from peristaltic to completely absent along with a decline in the LES pressure.

Our retrospective study included a larger sample of 102 patients with SSc. Our protocol with our patients with SSc has been to obtain a baseline ETS study at the initial evaluation, then on a yearly basis to monitor for $\mathrm{ED}$, regardless of the presence of symptoms. Twenty-one out of 22 of our patients with dcSSc had deterioration of their ETS study compared to 47 out of 80 patients with lcSSc. Fifteen patients with lcSSc had no change in their ETS study on sequential examination, and interestingly, 18 patients with lcSSc showed improvement on subsequent ETS evaluation. These data help to provide important prognostic information to patients with SSc, suggesting that the vast majority of patients with dcSSc will have deterioration in esophageal involvement, whereas esophageal involvement in patients with $1 \mathrm{cSSc}$ is not progressive in all cases. As patients with SSc are at risk of developing erosive esophagitis, Barrett's esophagus, and esophageal cancer as a consequence of GERD secondary to ED, the results also suggest that routine sequential monitoring for esophageal dysfunction should be performed in all patients with SSc.

Our study is limited by its retrospective design and the lack of a control group for comparison. Patients were followed for varying durations, and although an attempt was made to obtain yearly ETS studies, the number of ETS studies performed varied from patient to patient. Medications known to alter esophageal function, such as calcium channel blockers and metoclopramide, were not taken into consideration.

Esophageal abnormalities are frequently found in patients with SSc. ED appears to be more frequent and severe in patients with dcSSc than in patients with lcSSc. Although prior data suggest that autoantibody status, particularly the presence of anti-Scl-70 antibody, is associated with $\mathrm{ED}$, our results were not in accordance. $\mathrm{ED}$ is likely to deteriorate in most if not all patients with dcSSc, and may not be progressive in patients with lcSSc. Given the potential associated comorbidities, routine initial screening and serial monitoring for ED should be a part of the evaluation of patients with SSc. In this regard, ETS is a useful diagnostic tool to evaluate esophageal function and to monitor the severity of esophageal involvement in $\mathrm{SSc}^{5}$.

\section{REFERENCES}

1. Clements PJ, Becvar R, Drosos AA, Ghattas L, Gabrielli A. Assessment of gastrointestinal involvement. Clin Exp Rheumatol 2003;21:S15-8.

2. Sjögren RW. Gastrointestinal motility disorders in scleroderma. Arthritis Rheum 1994;37:1265-82.

3. Poirier T, Rankin M. Gastrointestinal manifestations of progressive systemic scleroderma based on a review of 364 cases. Am J Gastroenterol 1972;58:30-44.

4. Turner R, Lipshutz W, Miller W, Rittenberg G, Schumacher HR, Cohen S. Oesophageal dysfunction in collagen disease. Am J Med
Sci 1973;265:191-9.

5. Kaye SA, Siraj QH, Agnew J, Hilson A, Black CM. Detection of early asymptomatic esophageal dysfunction in systemic sclerosis using a new scintigraphy grading method. J Rheumatol 1996;23:297-301.

6. Forbes A, Marie I. Gastrointestinal complications: the most frequent internal complication of systemic sclerosis. Rheumatology 2009;48:iii36-9.

7. Wipff J, Allanore Y, Soussi F, Terris B, Abitbol V, Raymond J, et al. Prevalence of Barrett's esophagus in systemic sclerosis. Arthritis Rheum 2005;52:2882-8.

8. Katzka DA, Reynolds JC, Saul SH, Plotkin A, Lang CA, Ouyang A, et al. Barrett's metaplasia and adenocarcinoma of the esophagus in scleroderma. Am J Med 1987;82:46-52

9. Taillefer R, Jadliwalla M, Pellerin E, Lafontaine E, Duranceau A. Radionuclide esophageal transit study in detection of esophageal motor dysfunction: Comparison with motility studies (manometry). J Nucl Med 1990;31:1921-6.

10. Mughal MM, Marples M, Bancewicz J. Scintigraphic assessment of esophageal motility: What does it show and how reliable is it? Gut 1986;27:946-53.

11. Tatsch K, Voderholzer WA, Weiss MJ, Schrottle W, Hahn K. Re-appraisal of quantitative esophageal scintigraphy by optimizing results with ROC analyses. J Nucl Med 1996;37:1799-805.

12. Klein HA. Receiver operating characteristic surfaces for esophageal transit scintigraphy in systemic sclerosis [abstract]. Eur J Nucl Med 1991;16 Suppl:S31.

13. Edenbrandt L, Theander E, Hogstrom M, Scheja A, Akesson A, Palmer J. Esophageal scintigraphy in normal subjects and patients with systemic sclerosis. J Nucl Med 1995;36:1533-7.

14. Geatti O, Shapiro B, Fig LM, Fossaluzza V, Franzon R, De Vita S, et al. Radiolabelled semisolid test meal clearance in the evaluation of esophageal involvement in scleroderma and Sjogren's syndrome. Am J Physiol Imaging 1991;6:65-73.

15. Cozzi F, Zucchetta P, Durigon N, Marzola MC, Bullo A, Favaro M, et al. Esophageal dysmotility in scleroderma patients with different forms of disease and ANA patterns: A scintigraphic study in 100 cases. Reumatismo 2003;55:86-92

16. Baron M, Arzoumanian A. Radionuclide esophageal transit studies in progressive systemic sclerosis: An analysis of longitudinal data. J Rheumatol 1991;18:1837-40.

17. Dantas RO, Meneghelli UG, Oliveira RB, Villanova MG. Esophageal dysfunction does not always worsen in systemic sclerosis. Clin Gastroenterol 1993;17:281-5.

18. Subcommittee for Scleroderma Criteria of the American Rheumatism Association Diagnostic and Therapeutic Committee. Preliminary criteria for the classification of systemic sclerosis (scleroderma). Arthritis Rheum 1980;23:581-90.

19. Clements PJ, Lachenbruch PA, Ng SW, Simmons M, Sterz M, Furst DE. Skin score. A semiquantitative measure of cutaneous involvement that improves prediction of prognosis in systemic sclerosis. Arthritis Rheum 1990;33:1256-63.

20. Ntoumazios SK, Voulgari PV, Potsis K, Koutis E, Tsifetaki N, Assimakopoulos DA. Esophageal involvement in scleroderma: Gastroesophageal reflux, the common problem. Semin Arthritis Rheum 2006;36:173-81.

21. Lapadula G, Muolo P, Semeraro F, Covelli M, Brindicci D, Cuccorese G, et al. Esophageal motility disorders in the rheumatic diseases: A review of 150 patients. Clin Exp Rheumatol 1994;12:515-21.

22. Marie I, Dominique S, Levesque H, Ducrotte P, Denis P, Hellot MF, et al. Esophageal involvement and pulmonary manifestations in systemic sclerosis. Arthritis Rheum 2001;45:346-54.

23. Airò P, Della Casa D, Danieli E, Missale G, Cattaneo R, Cestari R. Oesophageal manometry in early and definite systemic sclerosis. 
Clin Rheumatol 2005;24:370-6.

24. Nakajima K, Hasegawa M, Inaki A, Wakabayashi H, Hosoya T, Takehara K, et al. Esophageal transit study using a sliding sum image: Application to patients with probable and definite systemic sclerosis. Ann Nucl Med 2011;25:325-31.

25. Nakajima K, Kawano M, Kinuya K, Sato S, Takehara K, Tonami N. The diagnostic value of oesophageal transit scintigraphy for evaluating the severity of oesophageal complications in systemic sclerosis. Nucl Med Commun 2004;25:375-81.
26. Davidson A, Russell C, Littlejohn GO. Assessment of esophageal abnormalities in progressive systemic sclerosis using radionuclide transit. J Rheumatol 1985;12:472-7.

27. Pakozdi A, Wilson H, Black CM, Denton CP. Does long term therapy with lansoprazole slow progression of oesophageal involvement in systemic sclerosis? Clin Exp Rheumatol 2009;3 Suppl 54:5-8. 